\title{
Genetic Basis and Function of Mating-Type Genes in Aspergillus cristatus
}

\author{
Ge $Y Y^{1}$, Yu FM ${ }^{1}$, Yang $Z J^{1}$, Tan $Y M^{2,3}$, Xiang $T^{1}$, Chen $X^{1}$, Yu $Y X^{1}$, Chen $L^{1}$, \\ Liu $\mathbf{Z Y}^{2 *}$
}

${ }^{1}$ College of Life and Science, Guizhou University, Guiyang 550025, Guizhou, China

${ }^{2}$ Guizhou Key Laboratory of Agriculture Biotechnology, Guiyang 550006, Guizhou, China

${ }^{3}$ Guizhou Academy of Agricultural Science, Guiyang 550006, Guizhou, China

Ge YY, Yu FM, Yang ZJ, Tan YM, Xiang T, Chen X, Yu YX, Chen L, Liu ZY 2019 - Genetic Basis and Function of Mating-Type Genes in Aspergillus cristatus. Mycosphere 10(1), 622-633, Doi 10.5943/mycosphere/10/1/12

\begin{abstract}
Aspergillus cristatus is a homothallic fungus, the genome of which contains two mating-type (MAT) genes, MAT1-1-1 and MAT1-2-1. Previous studies showed that the origin of MAT loci in this species differs from that of other fungi, and their functions have never been described. In this study, MAT1-1-1 and MAT1-2-1 were individually deleted by gene replacement, confirming that these genes regulate the sexual development of Aspergillus cristatus, with the two single-deletion mutants showing similar phenotypes. To explain this phenomenon, the evolutionary relationships of the core domains from high-mobility-group box (HMGB) domain, high-mobility-group (HMG) domain, and alpha $1(\alpha 1)$ domain were investigated, with the findings indicating that $\alpha 1$ domain and HMG domain have a close evolutionary relationship. Transcriptomic analyses indicated that mating-type genes regulate the sexual development of Aspergillus cristatus by controlling the expression levels of pheromone precursor and receptor genes. This paper presents the first systemic investigation of the function of MAT genes from Aspergillus cristatus.
\end{abstract}

Key words - Aspergillus cristatus - mating-type gene - sexual development

\section{Introduction}

Sexual development is a common, albeit not ubiquitous, mode of reproduction in fungi. In Ascomycota, sexual development involves cell fusion, nuclear fusion, and meiosis. These stages are orchestrated by the MAT locus, which encodes key transcription factor genes that govern identity and developmental fate (Lee et al. 2010, Dyer \& O’Gorman 2012, Krijgsheld et al. 2013). Heterothallic fungi have two different MAT locus genes, one encoding a protein with a highmobility-group (HMG) domain, called MAT1-2-1, and the other encoding a protein with an alpha box $(\alpha 1)$ domain, called MAT1-1-1. These two genes are assigned to different mating partners (Turgeon \& Yoder 2000, Kück \& Pöggeler 2009). In homothallic fungi, by contrast, the genome contains both MAT1-1-1 and MAT1-2-1 genes, so these species are self-fertile (Paoletti et al. 2007).

Aspergillus cristatus is a taxon that is dominant in the fermentation processes of Fuzhuan brick tea, and may produce abundant golden cleistothecia in later stages of the fermentation process (Tan et al. 2017). Among aspergilli, most species can reproduce exclusively asexually, but $A$. cristatus can undergo both sexual and asexual development under conditions of high and low 
osmolarity; the genome of this species has also been sequenced, which has facilitated the study of A. cristatus (Ge et al. 2016, 2017). Genome analyses have shown that the MAT locus genes of $A$. cristatus are located in scaffolds 5 and 6, respectively. Evolutionary analyses have also revealed that the MAT loci of A. cristatus differ for those of other Aspergillus species (Ge et al. 2016). Moreover, the function of MAT genes from A. cristatus has never been reported.

In this study, we used gene replacement to assess the function of MAT genes in A. cristatus. According to the evolutionary relationship of the core domains from HMGB, HMG, and $\alpha 1$, we explained why single-deletion mutants of MAT1-1-1 and MAT1-2-1 have similar phenotypes. Moreover, we analyzed the regulatory mechanism by which MAT1-1-1 and MAT1-2-1 control sexual development via transcriptome sequencing of the wild-type strain and MAT gene mutants. This paper reports for the first time the function and evolutionary relationship of MAT1-1-1 and MAT1-2-1 in A. cristatus, and also provides some important results for understanding the regulatory mechanisms of MAT genes.

\section{Materials \& Methods}

\section{Strains, growth conditions, and morphological observation}

Aspergillus cristatus E4 (CGMCC 7.193) was isolated from Fuzhuan brick tea manufactured by Yiyang Tea Factory Co. Ltd. (Yi Yang City, Hunan Province, China) and cultured on a cellulose membrane placed on MYA medium (malt extract, 20 g; yeast extract powder, 20 g; sucrose, 30 g; agar powder, $18 \mathrm{~g}$; and water, $1000 \mathrm{~mL}$ ) in the dark at $28{ }^{\circ} \mathrm{C}$ for 2 to 7 days. Mycelia were then fixed with $0.5 \% \mathrm{v} / \mathrm{v}$ glutaraldehyde in the dark at $4{ }^{\circ} \mathrm{C}$ for $12 \mathrm{~h}$, washed three times with $0.1 \mathrm{~mol} / \mathrm{L}$ PBS, fixed with $1 \%$ osmic acid for $2 \mathrm{~h}$, washed three times with $0.1 \mathrm{~mol} / \mathrm{L} \mathrm{PBS}$, dehydrated with 50\%-99\% thiobarbituric acid, freeze-dried under a vacuum, and coated with gold for morphological observation using a Hitachi S-3400N electron scanning microscope (Hitachi Corporation, Tokyo, Japan) (Ge et al. 2016). Moreover, mutant strains were cultured on MYA, low-osmolarity MYA (5\% $\mathrm{NaCl})$, and high-osmolarity MYA (17\% $\mathrm{NaCl})$, and the colony morphologies of wild-type and mutant strains were observed using an optical microscope (Olympus, Tokyo, Japan).

\section{Deletion of mating genes in Aspergillus cristatus}

Two vectors for the deletion of MAT1-1-1 (SI65_05562) and MAT1-2-1 (SI65_06277) (Ge et al. 2016) were constructed, aiming at inserting the hygromycin-resistance cassette into the coding region of the genes to be deleted (Sugui et al. 2005, Mullins et al. 2001, Huang et al. 2015). In brief, the 5' and 3' flanking sequences of mating-type genes were amplified using genomic DNA as a template with the primer pairs MAT1-1-1_UL and MAT1-1-1_UR (resulting in a fragment size of 930 bp), MAT1-1-1_DL and MAT1-1-1_DR (fragment size of 713 bp), MAT1-2-1_UL and MAT12-1_UR (fragment size of 1121 bp), and MAT1-2-1_DL and MAT1-2-1_DR (fragment size of 834 bp) (Table 1). The products were digested with the restriction enzymes Xho I, BamH I, Spe I, and Xba I, respectively, and then inserted into the corresponding sites of the binary vector pDHt for fungal transformation (Mullins et al. 2001). The mutants were verified by Southern blotting with a probe (Hyg-p) and PCR with the primers MAT1-1-1_S and MAT1-2-1_S (Table 1).

Table 1 Primers used for PCR amplification for mating-type genes deletion and detection

\begin{tabular}{llc}
\hline Primer names & Primer sequences & $\begin{array}{c}\text { Products size } \\
\text { (bp) }\end{array}$ \\
\hline MAT1-1-1_UL & 5'-CTCGAGCGACTTTCGCATCACTCC-3' & 930 \\
MAT1-1-1_UR & 5'-GGATCCCCTACCCCATCACCCAT-3' & 713 \\
MAT1-1-1_DL & 5'-ACTAGTAAATGGGCTATCCTTGC-3' & \multirow{2}{*}{1121} \\
MAT1-1-1_DR & 5'-TCTAGAATGGCTTGTATCGTCCC-3' & \\
MAT1-2-1_UL & 5'-CTCGAGTGGAGATAGAATCCGAAACG-3' & \\
MAT1-2-1_UR & 5'-GGATCCACAGATACCGCAGAGCC-3' & \\
\hline
\end{tabular}


Table 1 Continued.

\begin{tabular}{llc}
\hline Primer names & Primer sequences & $\begin{array}{c}\text { Products size } \\
\text { (bp) }\end{array}$ \\
\hline MAT1-2-1_DL & 5'-ACTAGTGCTTCATTCGGTTCGTC-3' & 834 \\
MAT1-2-1_DR & 5'-TCTAGAAGCCCAAGTCAATCTCG-3' & \\
MAT1-1-1_SL & 5'-TCCGTCTGAATGAAGCC-3' & 3158 \\
MAT1-1-1_SR & 5'-GGGCGGCATAGAAGTGT-3' & \\
MAT1-2-1_SL & 5'-AAGGCTTTCTCGGTTTG-3' & 2814 \\
MAT1-2-1_SR & 5'-ACCGTTCCGTAGTTGCT-3' & \\
Hyg-pL & 5'-TTCGATGTAGGAGGGCGTGGAT-3' & 626 \\
Hyg-pR & 5'-CGCGTCTGCTGCTCCATACAAG -3' & \\
\hline
\end{tabular}

\section{MAT gene sequence acquisition and analysis}

We analyzed the promoters, terminators, and introns of MAT genes using Fgenesh ${ }^{+}$(Solovyev et al. 2006). The high-mobility-group box (HMGB), HMG, and alpha $1(\alpha 1)$ amino acid sequences from aspergilli retrieved from AspGD (Aspergillus Genome Database) and NCBI (National Center for Biotechnology Information) are reported in Additional file 1. The core regions of these sequences were revealed using Kalign (Lassmann \& Sonnhammer 2006) and datasets were analyzed with WebLogo (Crooks et al. 2004). Sequence alignments were generated with Clustal W2 (Larkin et al. 2007). The alignment results were used to construct a phylogenetic tree using maximum likelihood by MEGA 7.0 (Kumar et al. 2016). Moreover, secondary structures of MAT gene sequences were predicted by Jpred 4 (Drozdetskiy et al. 2015). Fold recognition and 3D structure prediction were performed with Phyre 2 (Kelley et al. 2015).

\section{RNA extraction and transcriptome sequencing}

Mycelia of three different strains, namely, A. cristatus E4 (wild type), MAT1-1-1 deletion

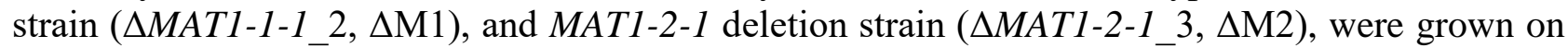
MYA for $40 \mathrm{~h}$ (ascogonial coils begin to form; stage 1) and $48 \mathrm{~h}$ (cleistothecia can be observed and enumerated; stage 2). For the culture at each stage, three independent biological replicates were designed; thus, total RNA was extracted from a total of 18 samples using TRIzol reagent, in accordance with the manufacturer's instructions (Life Technologies Co., Ltd., Carlsbad, CA, USA). RNA was quantified using a NanoDrop 2000 UV-vis spectrophotometer (Thermo Fisher Scientific Inc., Boston, MA, USA) based on the absorbance at 260 and $280 \mathrm{~nm}$. A total of $1 \mu \mathrm{g}$ of RNA per sample was used as a template for the construction of cDNA libraries. The prepared libraries were sequenced on a BGISEQ-500 RNA-ref platform (BGI, Shenzhen, China), and 100-bp pair end (PE) reads were generated.

\section{Identification and characterization of differentially expressed genes}

The high-quality clean reads were mapped to the genome and gene dataset of A. cristatus E4 by HISAT and Bowtie 2 (Kim et al. 2015), and then the expression levels of the transcripts were calculated by RSEM (Li \& Dewey 2011). In this study, an absolute $\log _{2}$ value (fold change) $\geq 1$ and adjusted $\mathrm{P}$ value $\leq 0.05$ were used as threshold values to screen for differentially expressed genes (DEGs). All data contributing to this study have been deposited with the NCBI under BioProject PRJNA431929. The RNA-seq expression dataset has been deposited at the NCBI's Sequence Read Archive under accession code SRP131629.

\section{Results}

\section{Analysis of MAT gene sequences}

In this study, we used Fgenesh to analyze promoters, terminators, and introns of MAT1-1-1 (SI65_05562) and MAT1-2-1 (SI65_06277) from the A. cristatus genome (Ge et al. 2016, Solovyev et al. 2006). The results indicate that MAT1-1-1 of A. cristatus is 1209 bases long and has one 
intron of $62 \mathrm{bp}$, and encodes a protein with 384 amino acids. MAT1-2-1 of A. cristatus is 1188 bases long, has two introns of 63 and 51 bp each, and encodes a protein with 357 amino acids.

\section{Functional characterization of mating-type genes}

MAT1-1-1 and MAT1-2-1 of A. cristatus were individually deleted by gene replacement. A total of four MAT1-1-1 and two MAT1-2-1 deletion mutants were obtained, all of which showed

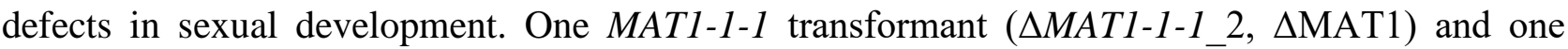
MAT1-2-1 transformant ( $\left.\triangle M A T 1-2-1 \_3, \triangle M A T 2\right)$ were chosen for further study. Subsequently, we observed the colony morphology of the wild-type strain and mutants. On MYA, the colonies of the mutant strains were lighter yellow and larger than for the control strain; moreover, the black exudate from the colony surface of wild-type strains was more extensive than that from the mutants (Fig. 1; A1, B1 and C1). On MYA with 5\% NaCl, the colonies of the mutant strains and the wildtype strain were nearly the same size, and these cultures did not produce exudate; the color of the colonies of the mutant strains was lighter than that of the control strain (Fig. 1; A2, B2 and C2). The colony morphologies of the three strains were similar on MYA with $17 \% \mathrm{NaCl}$ (Fig. 1; A3, B3 and C3).

The cleistothecium and ascospore morphology of the mutants and wild-type strains was observed by electron scanning microscopy. The results showed that two mutants produced fewer cleistothecia than the wild type under conditions of sexual development (Fig. 1; A4, B4 and C4). Moreover, the developed cleistothecia were immature and smaller than those in the wild-type strain (Fig. 1; A5, B5 and C5). Notably, no ascospores formed in the cleistothecia of the mutants (Fig. 1; A6, B6 and C6). This indicates that either of the MAT genes is essential for ascospore production and for the sexual development of $A$. cristatus.

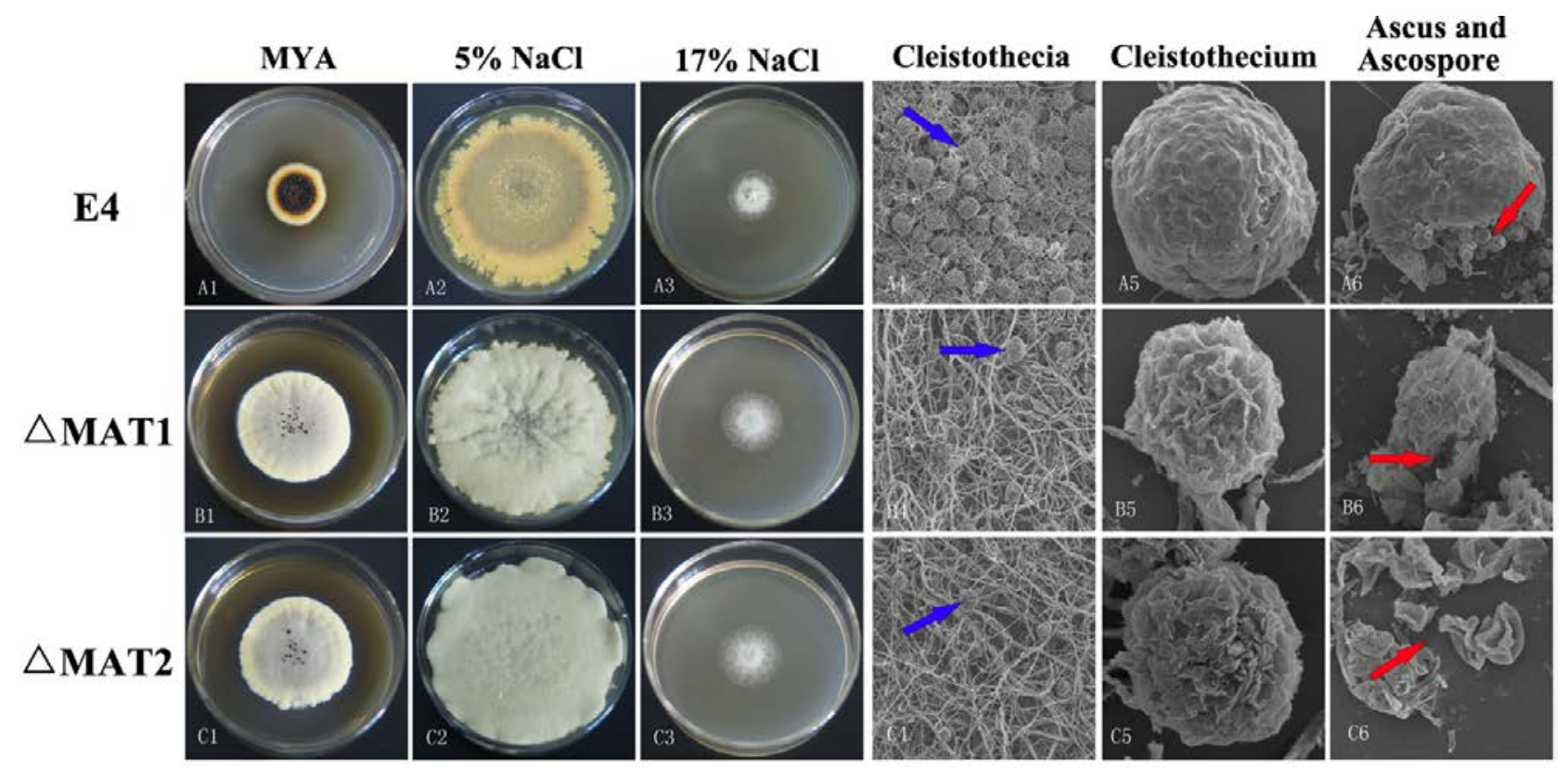

Figure 1 - Phenotypic observation of the MAT mutants and wild-type strains under different growth conditions. A1-A6 show the results for wild-type strain Aspergillus cristatus E4. B1-B6 show the results for MAT1-1-1 mutant. C1-C6 show the results for MAT1-2-1 mutant. Blue arrows indicate cleistothecia on the mycelium, red arrows indicate the ascus and ascospore. In Aspergillus cristatus, E4 has ascus and ascospore, while MAT1-1-1 and MAT1-2-1 mutants have no ascus and ascospore.

Since the phenotypes of MAT1-1-1 and MAT1-2-1 mutants were similar, we speculated that the MAT genes from $A$. cristatus share similar functions. To confirm this, we performed an analysis of the evolution of the mating-type genes. 


\section{Evolutionary analysis of mating-type genes}

We selected protein sequences of aspergilli from AspGD and the NCBI database (Additional file 1) and divided them into three datasets, namely, HMGB domain, $\alpha 1$ domain, and HMG domain datasets. Subsequently, we obtained the core domains of the three datasets using Kalign (Lassmann \& Sonnhammer 2006); the core domain of $\alpha 1$ contained 41 amino acids and the core domains of HMG and HMGB contained 43 amino acids. The correlation of these amino acid sequences was derived using Weblogo (Fig. 2a) (Crooks et al. 2004), and the results revealed higher similarity between HMG and $\alpha 1$ domains than with the HMGB domain (Fig. 2b). Meanwhile, a maximum likelihood phylogram was constructed using the core domains of three datasets. The results showed that the sequences of the three domains were clustered into two clades. HMGB domain sequences were distributed in a monophyletic clade, while the HMG and $\alpha 1$ domains were mostly distributed in another clade (Fig. 3). These results also indicated that HMG and $\alpha 1$ domain sequences have closer affinity than the HMGB domain sequence.

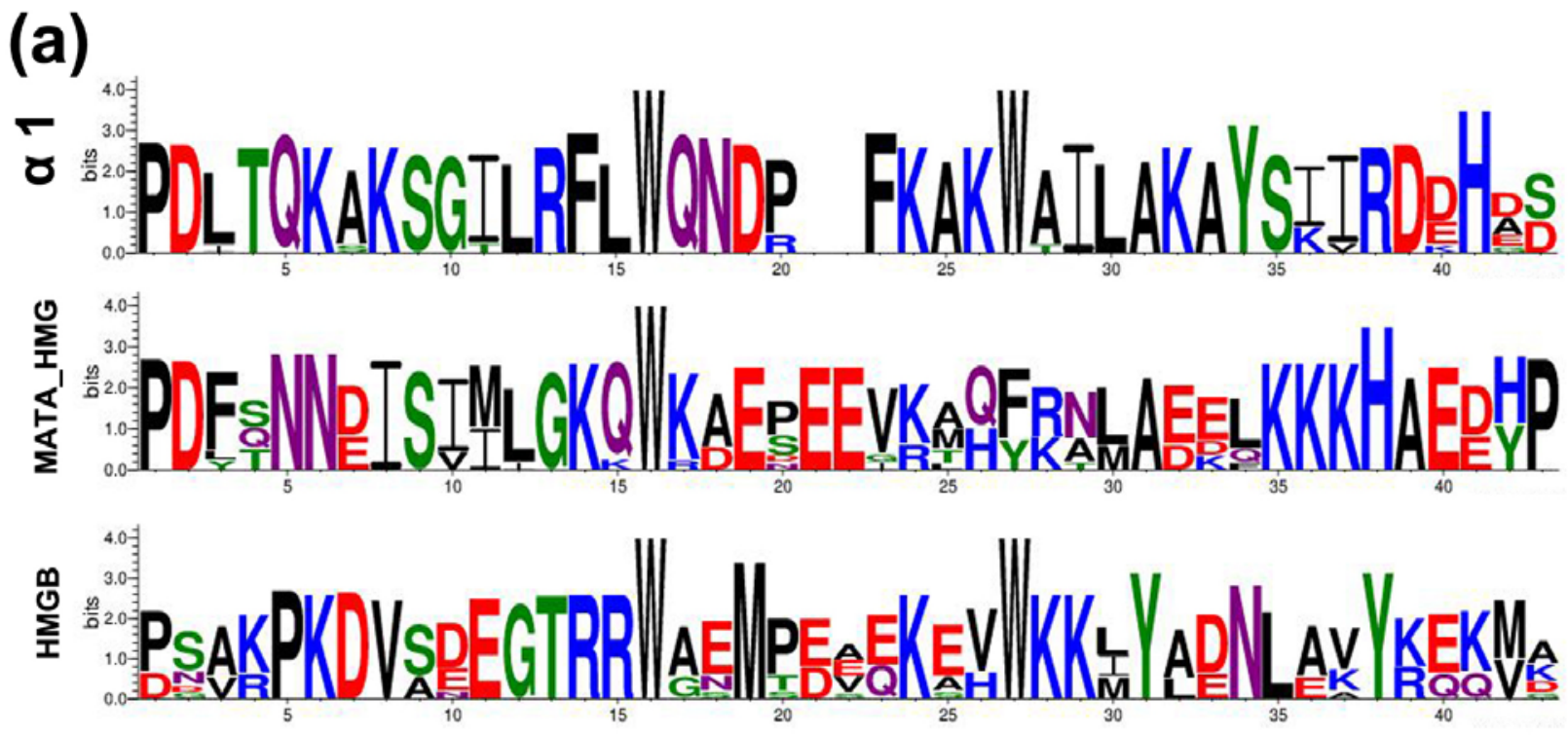

\section{(b)}

\section{$\alpha 1$ PDLTQKAKSGILRFLWQNDP--FKAKWAILAKAYSKIRDEHDS MATA PDFQNNDISIMLGKQWKAEPEEVRAHYKALADEQKKKHAEEYP HMGB DGVKPKDVAEEGTRRWGNMSDVEKSVWKKIYLENLEKYRQQMD}

Figure 2 - Conserved sequence of HMGB, MAT_HMG and $\alpha 1$ domains. a Weblogo representation of a conserve sequence in $\alpha 1$, MATA_HMG and HMGB. The X-axis represents amino acid position from the $\mathrm{N}$ to $\mathrm{C}$ terminal. The height of each letter within the stack reflects the relative frequency of the corresponding amino acid at the position, the overall height of each letter indicates the sequence conservation at the position (measured in bits). b Consensus core sequences of conserved amino acid residues.

The sequence conservation between HMG and $\alpha 1$ domains suggested that they may have similar secondary and tertiary structures. As such, we first predicted the secondary structures of HMG and $\alpha 1$ domains using Jpred 4 (Drozdetskiy et al. 2015). The results showed that the HMG and $\alpha 1$ domains form three helixes (Fig. 4a). Helix 1 of the $\alpha 1$ domain is four residues shorter than that of the HMG domain, and helix 3 of the $\alpha 1$ domain is three residues shorter than that of the HMG domain (Fig. 4b, c). Protein sequences used for secondary structure prediction were submitted to Phyre 2 for fold recognition (Kelley et al. 2015). As expected, the best matching templates for the $\alpha 1$ domain of $A$. cristatus were known HMG-box template structures. The 
likelihood of the homology was high (99\%) and all tested mating-type proteins had the HMG-box family fold descriptor. Thus, the $\alpha 1$ and HMG domains of A. cristatus have the same genealogy. According to these results, we speculated that MAT1-1-1 (possessing an $\alpha 1$ domain) and MAT1-2-1 (possessing HMG domains) of A. cristatus have the same origin.

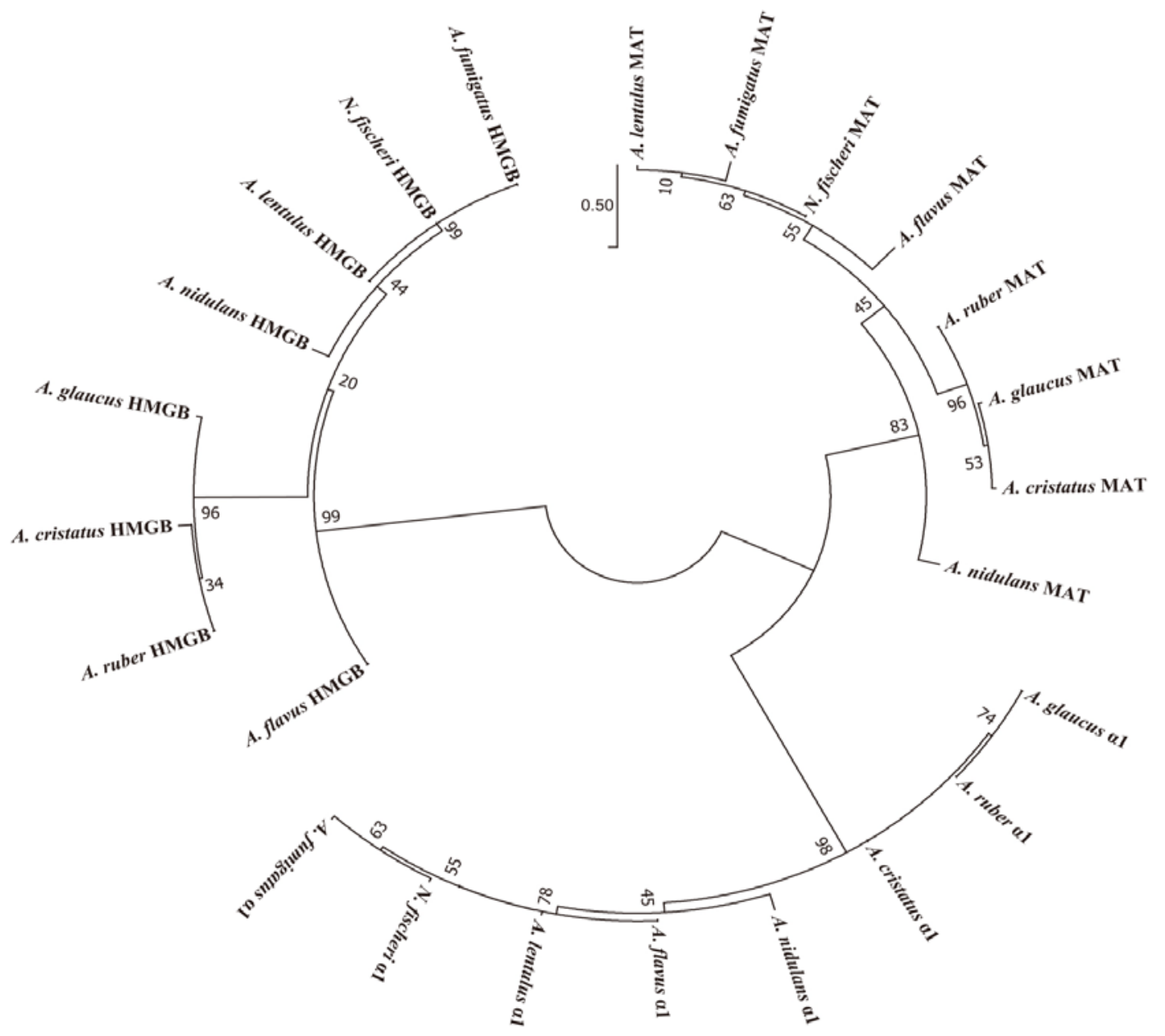

Figure 3 - Unrooted phylogram for the HMGB, MAT_HMG and the $\alpha 1$ domain core amino acid sequences. Clustering of core amino acid sequences using maximum-likelihood from MEGA 7.0 (Kumar et al. 2016), 1000 bootstrap replicates were used. Three domains clustered in two clades, with HMGB domain sequences distributed in a monophyletic clade, and MAT_HMG and $\alpha 1$ domains mostly distributed in a clade. Abbreviations: HMGB, high mobility group box; MAT, MAT_HMG; $\alpha 1$, alpha 1.

\section{Mechanism of regulation of sexual development by mating-type genes}

This study identified 551 differentially expressed genes by comparing gene expression levels from the mutants and the wild-type strain, accounting for $5.44 \%$ of the total number of genes (Additional file 2). Of these, 116 and 262 genes were the differentially expressed genes from $\triangle$ MAT1 at stage 1 and stage 2, respectively. 154 and 257 genes were the differentially expressed genes from $\triangle \mathrm{MAT2}$ at stage 1 and stage 2, respectively (Fig. 5). Among these genes, 9 differentially expressed genes are associated with sexual reproduction of $A$. cristatus (Table 2; Fig. 6). The expression levels of ppgA and preA from $\triangle \mathrm{MAT} 1$ were down-regulated in both stages, while $p r e B$, ste 14 , rce 1 , and $f p h A$ of $\triangle \mathrm{MAT} 1$ were only down-regulated at stage 1 , and $g p r K, f l b D$, and $f l b A$ were only down-regulated at stage 2 . Meanwhile, preB, ste 14 , and rce 1 of $\triangle \mathrm{MAT} 2$ were down-regulated at both stages, while $g p r K$ and $f l b D$ of $\triangle$ MAT2 were only down-regulated at stage 2 , but ppgA of $\triangle \mathrm{MAT} 2$ was up-regulated at stage 2. Moreover, $f l b D, p p g A$, rce1, ste14, and gprK 
were regulated together by MAT1-1-1 and MAT1-2-1, while other genes were only regulated by one of the MAT genes. These results suggest that MAT1-1-1 and MAT1-2-1 regulate the sexual development of $A$. cristatus by controlling genes for pheromone formation and signaling, and by controlling genes for cleistothecium development. The results also indicate that sporulation-related genes regulated by MAT1-1-1 differ from those regulated by MAT1-2-1.

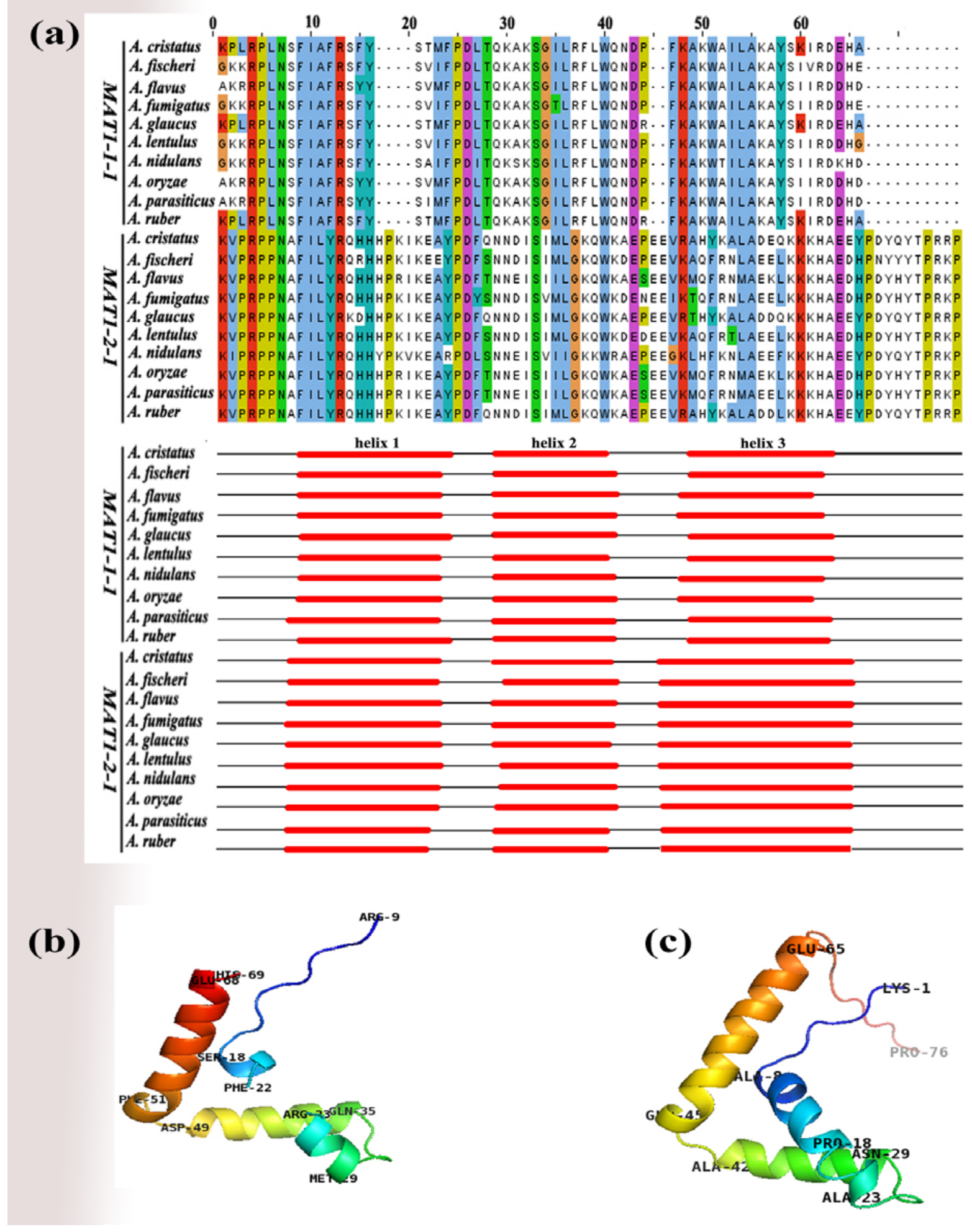

Figure 4 - Predicted secondary and 3D structures of the $\alpha 1$ and MAT_HMG domains from Aspergillus cristatus. a Secondary structure of MAT_HMG and the $\alpha \overline{1}$ domain from other aspergilli. b in silico 3D structure model of the $\alpha 1$ domain from MAT1-1-1 in Aspergillus cristatus. c in silico 3D structure of the MAT_HMG domain from MAT1-2-1 in Aspergillus cristatus. 


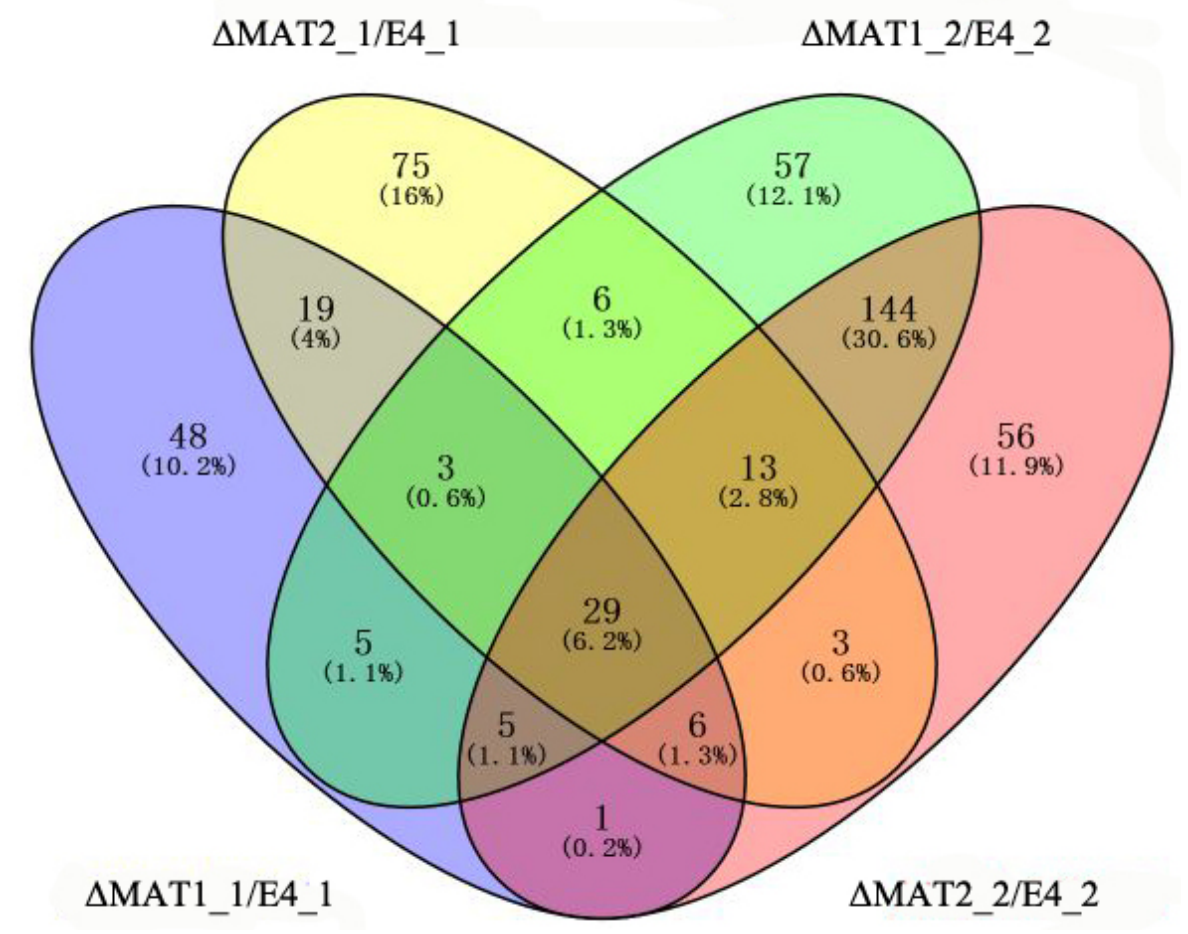

Figure 5 Comparison of the differentially expressed genes from the wild strain and MAT genes mutants. In the figure, $\triangle \mathrm{MAT} 1 \mathrm{~B} / \mathrm{E} 4 \mathrm{1}$ indicates the differentially expressed genes from the MAT1-1-1 deletion strain relative to Aspergillus cristatus E4 at stage 1, while $\triangle$ MAT1_2/E4_2 indicates that at stage 2 . The results of $\triangle \mathrm{MAT} 2 \_1 / \mathrm{E} 4 \_1$ and $\triangle \mathrm{MAT} 2 \_2 / \mathrm{E} 4 \_2$ are similar to those described previously.

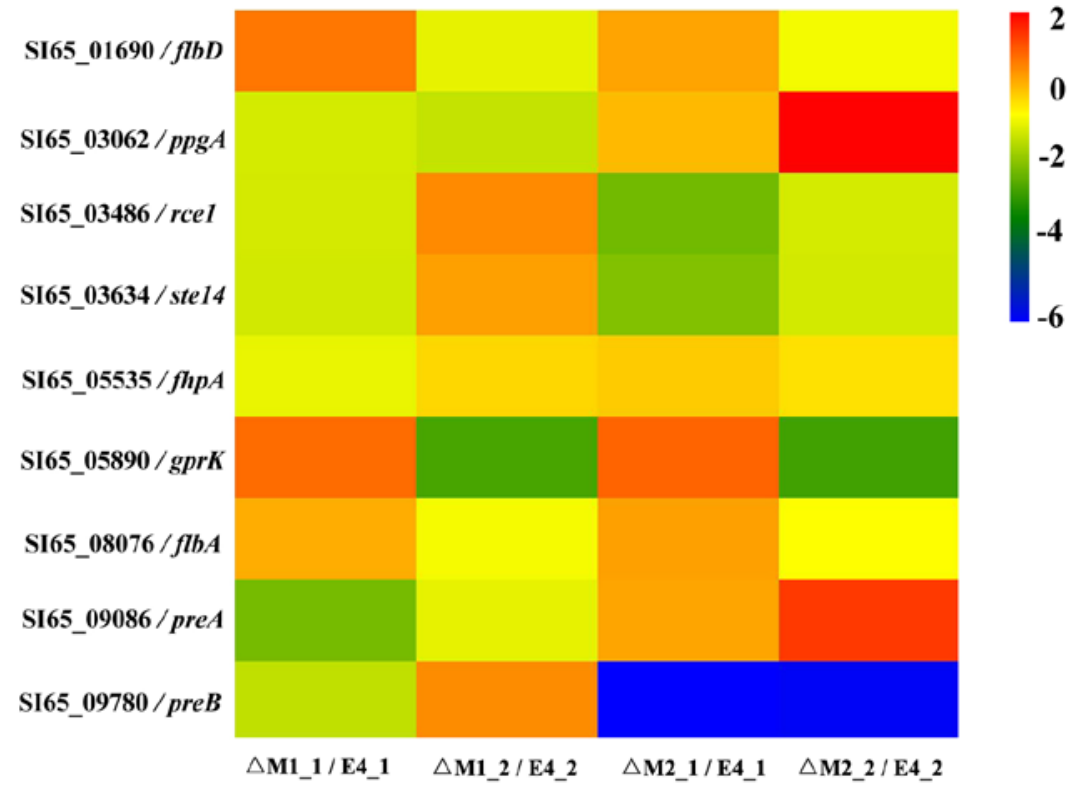

Figure 6 Differentially expressed genes from MAT1-1-1 and MAT 1-2-1 mutants. In the figure, changes in background color from blue to red indicate changes in log2 change fold values $(-6,-4$, $-2,0$, and 2; upper right) of gene expression levels under conditions of $40 \mathrm{~h}$ and $48 \mathrm{~h}$ of growth on MYA. The codes on the right, such as SI65_09086/preA, are gene IDs or names. Sample information is detailed beneath the images. $\triangle$ MAT1_1/E4_1 indicates $\log 2$ fold change of the gene expression level of the MAT1-1-1 deletion strain relative to Aspergillus cristatus E4 at stage 1, while $\triangle \mathrm{MAT} 12 / \mathrm{E} 4 \_2$ indicates that at stage 2 . The results of $\triangle \mathrm{MAT} 2 \_1 / \mathrm{E} 4 \_1$ and $\Delta$ MAT2_2/E4_2 are similar to those described previously. 
Table 2 Differentially expressed genes associated with sexual reproduction of Aspergillus cristatus in MAT1-1-1 and MAT1-2-1 deletion-mutants.

\begin{tabular}{|c|c|c|c|c|c|c|c|}
\hline $\begin{array}{l}\text { Genes } \\
\text { names }\end{array}$ & $\begin{array}{l}\text { AspGD } \\
\text { ID }\end{array}$ & A. cristatus & Function & $\begin{array}{l}\text { Log2 Fold } \\
\text { Change } \\
\left(\triangle M A T 1-1-1 \_1\right. \\
\text { / E4-1) } \\
\end{array}$ & 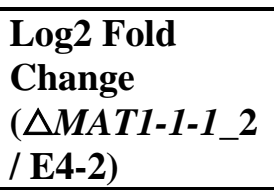 & $\begin{array}{l}\text { Log2 Fold } \\
\text { Change } \\
\left(\triangle M A T 1-2-1 \_1 /\right. \\
\text { E4-1) }\end{array}$ & 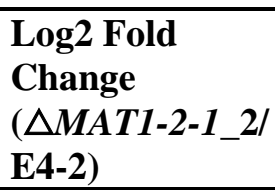 \\
\hline$f l b D$ & AN0279 & SI65_01690 & $\begin{array}{l}\text { Regulation of conidiophore } \\
\text { formation }\end{array}$ & 0.327948906 & -1.17857348 & -0.09443022 & -1.03561797 \\
\hline $\operatorname{ppg} A$ & AN5791 & SI65_03062 & Pheromone precursor & -1.33098067 & -1.48248346 & -0.31501377 & 1.413918246 \\
\hline rce1 & AN6528 & SI65_03486 & $\begin{array}{l}\text { Role in pheromone precursor } \\
\text { processing }\end{array}$ & -1.34618001 & 0.152754026 & -2.26236605 & -1.33090013 \\
\hline ste14 & AN6162 & SI65_03634 & $\begin{array}{l}\text { Role in pheromone precursor } \\
\text { processing }\end{array}$ & -1.36356792 & -0.05119989 & -2.09475117 & -1.35388858 \\
\hline fhpA & AN4521 & SI65_05535 & Regulation of sexual development & -1.13870613 & -0.56537125 & -0.44966608 & -0.65871797 \\
\hline gprK & AN7795 & SI65_05890 & $\begin{array}{l}\text { Putative heterotrimeric G-protein } \\
\text { coupled receptor component }\end{array}$ & 0.426691541 & -2.63516061 & 0.492665098 & -2.70359253 \\
\hline$f l b A$ & AN5893 & SI65_08076 & $\begin{array}{l}\text { Inhibition of vegetative growth } \\
\text { regulation Homothallic cleistothecia } \\
\text { formation }\end{array}$ & -0.18121976 & -1.00900537 & -0.05402233 & -0.92864768 \\
\hline preA & AN7743 & SI65_09086 & $\begin{array}{l}\text { G-protein coupled pheromone } \\
\text { receptor }\end{array}$ & -2.2166909 & -1.17938831 & -0.10736702 & 0.88184842 \\
\hline preB & AN2520 & SI65_09780 & $\begin{array}{l}\text { G-protein coupled pheromone } \\
\text { receptor }\end{array}$ & -1.5221798 & 0.123230336 & -5.6924923 & -5.61106 \\
\hline
\end{tabular}

\section{Discussion}

Mating-type genes are pivotal in the sexual development of fungi. In Aspergillus nidulans, it has previously been shown that deletion of either of the MAT genes resulted in the significant decreased production of cleistothecia, which are also small and entirely sterile (Paoletti et al. 2007). In this study, MAT gene mutants could not generate ascospores and failed to produce mature cleistothecia. These results are consistent with the reported function of MAT genes from A. nidulans (Paoletti et al. 2007). However, we also observed the interesting phenomenon that $\triangle \mathrm{MAT1}$ and $\triangle \mathrm{MAT} 2$ deletion mutants of $A$. cristatus showed similar phenotypes. Thus, we further studied the mechanism behind this phenomenon by investigating the evolutionary relationship of mating-type genes.

In the literature on studies of MAT genes, there are reports about the evolutionary origin of HMG-domain proteins and $\alpha 1$-domain proteins (Idnurm et al. 2008). For example, Martin researched the evolutionary relationship of the HMG domain and $\alpha 1$ domain from representative species from major groups of Ascomycota and proposed that extant $\alpha 1$-box genes originated from an ancestral HMG gene (Martin et al. 2010). However, the genealogical analysis of MAT1-1-1 and MAT1-2-1 from Ulocladium indicated separate evolutionary events for the two MAT genes (Geng et al. 2014). The phylogenetic relationship of MAT1-1-1 and MAT1-2-1 from A. cristatus was analyzed using the conserved domain sequence of the corresponding 
protein products. The results indicated that the $\alpha 1$ domain from MAT1-1-1 and the HMG domain from MAT1-2-1 have a close relationship, and the two genes could have originated from a single ancestral HMG gene. These results are consistent with representative species of Pezizomycotina (Martin et al. 2010).

We detected the change of expression levels of sporulation-related genes from the mutants by transcriptomic sequencing. The results showed that there are nine differentially expressed genes putatively involved in sexual development. Among these genes, $f l b D$ and $f p h A$ are associated with the regulation of sexual development (Purschwitz et al. 2008, Krijgsheld et al. 2013, ArratiaQuijada et al. 2012), and the genes ppgA, preA, preB, gprK, flbA, rce1, and ste14 are related to pheromone formation, transport, and sensing (Marr et al. 1990, Han et al. 2001, Pöggeler 2002, Seo et al. 2004, Yu 2006, Dyer \& O’Gorman 2012). Moreover, the results from Penicillium chrysogenum showed that MAT1-1-1 is related to ppg1 (ppgA, in aspergilli) and pre1 (preA, in aspergilli) (Becker et al. 2015). In A. cristatus, ppgA, preA, and preB are downregulated in $\triangle \mathrm{MAT} 1$ and $\triangle$ MAT2, indicating that mating-type genes are related to these genes. The results are consistent with previous reports on A. nidulans and P. chrysogenum (Paoletti et al. 2007, Becker et al. 2015).

In conclusion, the mating-type genes in A. cristatus are key for controlling sexual development. Mutants with the deletion of mating-type genes cannot form mature cleistothecia and ascospores. MAT1-1-1 and MAT1-2-1 in A. cristatus have a close evolutionary relationship. Notably, this paper reports for the first time the function of mating-type genes from A. cristatus and provides a valuable reference for research on the function of mating-type genes of fungi. However, the relationship between mating-type genes and other genes in A. cristatus remains to be clarified.

\section{Acknowledgements}

This work was supported by the National Natural Science Foundation Program of PR China (NSFC31660021; NSFC31860022); Guizhou Province Fund for Application Basic Research (GSTD-LH-[2015]-7683), Guizhou Province Platform for Innovative Talent Team ([2016]-5624), Talent Introduction Science Projects of Guizhou University [2016-(37)], and Guizhou Province Talent Platform ([2017]-5788), Construction Program of Biology First-class Discipline in Guizhou(GNYL[2017]-009).

We thank Professor Chengshu Wang (Institute of Plant Physiology \& Ecology, Shanghai Institute for Biology Science, Chinese Academy of Sciences) for providing the pDHt vector. We would like to express our gratitude to Professor Xingzhong Liu (State Key Laboratory of Mycology, Institute of Microbiology, Chinese Academy of Sciences) for discussions and critical reviewing of the manuscript.

\section{References}

Arratia-Quijada J, Sánchez O, Scazzocchio C, Jesús A. 2012 - FlbD, a Myb Transcription factor of Aspergillus nidulans, is uniquely involved in both asexual and sexual differentiation. Eukaryotic Cell 11(9), 1132-1142.

Becker K, Beer C, Frertag M, Kück U. 2015 - Genome-wide identification of target genes of a mating-type $\alpha$-domain transcription factor reveals functions beyond sexual development. Molecular Microbiology 95(6), 1002-1022.

Crooks GE, Hon G, Chandonia JM, Brenner SE. 2004 - WebLogo: A sequence logo generator. Genome Research 14(6), 1188-1190.

Drozdetskiy A, Cole C, Procter J, Barton GJ. 2015 - JPred4: a protein secondary structure prediction server. Nucleic Acids Research 43(W1), W389-394.

Dyer PS, O’Gorman CM. 2012 - Sexual development and cryptic sexuality in fungi: insights from Aspergillus species. FEMS Microbiology Reviews 36(1), 165-192.

Ge YY, Wang YC, Liu YX, Tan YM et al. 2016 - Comparative genomic and transcriptomic analyses of the Fuzhuan brick tea fermentation fungus Aspergillus cristatus. BMC Genomics 17, 428-440. 
Ge YY, Yu FM, Tan YM, Zhang XL et al. 2017 - Comparative transcriptome sequence analysis of sporulation-related genes of Aspergillus cristatus in response to low and high osmolarity. Current Microbiology 74, 806-814.

Geng Y, Li Z, Xia LY, Wang Q et al. 2014 - Characterization and phylogenetic analysis of the mating-type loci in the asexual ascomycete genus Ulocladium. Mycologia 106(4), 649-665.

Han KH, Han KY, Yu JH, Chae KS et al. 2001 - The $n s d D$ gene encodes a putative GATA-type transcription factor necessary for sexual development of Aspergillus nidulans. Molecular Microbiology 41(2), 299-309.

Huang W, Shang YF, Chen P, Gao Q et al. 2015 - MrpacC regulates sporulation, insect cuticle penetration and immune evasion in Metarhizium robertsii. Environmental Microbiology 17(4), 994-1008.

Idnurm A, Wolton FJ, Floyd A, Heitman J. 2008 - Identification of the sex genes in an early diverged fungus. Nature 451(7175), 193-196.

Kelley LA, Mezulis S, Yates CM, Wass MN et al. 2015 - The Phyre2 web portal for protein modeling, prediction and analysis. Nature Protocol 10(6), 845-858.

Kim D, Langmead B, Salzberg SL. 2015 - HISAT: a fast spliced aligner with low memory requirements. Nature Methods 12(4), 357-360.

Krijgsheld P, Bleichrodt R, Veluw GJ, Wang F et al. 2013 - Development in Aspergillus. Studies in Mycology 74, 1-29.

Kumar S, Stecher G, Tamura K. 2016 - MEGA7: Molecular Evolutionary Genetics Analysis Version 7.0 for Bigger Datasets. Molecular Biology and Evolution 33(7), 1870-1874.

Kück U, Pöggeler S. 2009 - Cryptic sex in fungi. Fungal Biology Reviews 23, 86-90.

Larkin MA, Blackshields G, Brown NP, Chenna R et al. 2007 - Clustal W and Clustal X version 2.0. Bioinformatics 23, 2947-2948.

Lassmann T, Sonnhammer ELL. 2006 - Kalign, Kalignvu and Mumsa: web servers for multiple sequence alignment. Nucleic Acids Research 34, W596-W599.

Lee SC, Ni M, Li WJ, Shertz C et al. 2010 - The evolution of sex: a perspective from the fungal kingdom. Microbiology Molecular Biology Reviews 74(2), 298-333.

Li B, Dewey CN. 2011 - RSEM: accurate transcript quantification from RNA-Seq data with or without a reference genome. BMC Bioinformatics 12(1), 323.

Marr RS, Blair LC, Thornor J. 1990 - Saccharomyces cerevisiae STE14 gene is required for $\mathrm{COOH}$-terminal methylation of a-factor mating pheromone. Journal of Biological Chemistry 265(33), 20057-20060.

Martin T, Lu SW, Van Tilbeurgh, Ripoll DR et al. 2010 - Tracing the origin of the fungal a1 domain places its ancestor in the HMG-Box superfamily: implication for fungal Mating-Type evolution. PLoS One 5(12), 1-13.

Mullins ED, Chen X, Romaine P, Raina R et al. 2001 - Agrobacterium-mediated transformation of Fusarium oxysporum: An efficient tool for insertional mutagenesis and gene transfer. Phytopathology 91(2), 173-180.

Paoletti M, Seymour F, Alcocer M, Kaur N et al. 2007 - Mating type and the genetic basis of selffertility in the model fungus Aspergillus nidulans. Current Biology 17, 1384-1389.

Pöggeler S. 2002 - Genomic evidence for mating abilities in the asexual pathogen Aspergillus fumigatus. Current Genetics 42, 153-160.

Purschwitz J, Müller S, Kastner C, Schoser M et al. 2008 - Functional and physical interaction of blue- and red-light sensors in Aspergillus nidulans. Current Biology 18(4), 255-259.

Seo JA, Han KH, Yu JH. 2004 - The gprA and gprB genes encode putative G protein coupled receptors required for self-fertilization in Aspergillus nidulans. Molecular Microbiology 53(6), 1611-1623.

Solovyev V, Kosarev P, Seledsov I, Vorobyev D et al. 2006 - Automatic annotation of eukaryotic genes, pseudogenes and promoters. Genome Biology 7(Suppl I), S10. 
Sugui JA, Chang YC, Kwon-Chung KJ. 2005 - Agrobacterium tumefaciens-mediated transformation of Aspergillus fumigatus: an efficient tool for insertional mutagenesis and targeted gene disruption. Applied and Environmental Microbiology 71(4), 1798-1802.

Tan YM, Wang YP, Ge YY, Ren XX et al. 2017 - Isolation and molecular identification of Aspergillus cristatus in fermented "fuzhuan" brick tea from Guizhou Province. Mycosystema 36(2), 154-163. (in Chinese)

Turgeon BG, Yoder OC. 2000 - Proposed nomenclature for mating type genes of filamentous Ascomycetes. 31, 1-5.

Yu JH. 2006 - Heterotrimeric G protein signaling and RGSs in Aspergillus nidulans. Journal of Microbiology 44(2), 145-154. 